\title{
Bilan et traitement de la maladie variqueuse
}

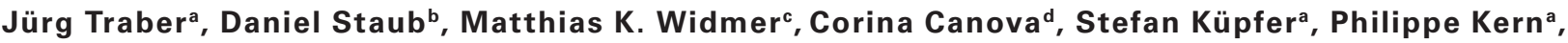 \\ Paolo Cassina ${ }^{a}$, Dominik Heima ${ }^{a}$ Nicolas Ducrey ${ }^{a}$, Rosmarie Holzingera, Christina Jeanneret ${ }^{a}$, Jürg Hafner $^{a}$
}

Membre du Comité directeur de la Société suisse de phlébologie (SSP); ' Président de I'Union des sociétés suisses des maladies vasculaires (USSMV);

${ }^{\circ}$ Président de la Société suisse de chirurgie vasculaire (SSCV); d Présidente de la Société suisse d'angiologie (SSA)

\section{Introduction}

La maladie variqueuse est une maladie très répandue du système veineux superficiel [1, 2]. Non traitée, elle progresse et entraîne des maladies secondaires et des complications importantes. Le traitement des troncs saphéniens variqueux et de leurs branches latérales constitue toujours une indication médicale. L'ablation des veines réticulaires et des télangiectasies (varicosités en toile d'araignée) est, quant à elle, principalement motivée pour des raisons esthétiques [3]. Le but de cette prise de position est de démontrer l'importance de la maladie variqueuse et des options thérapeutiques remboursables qui en découlent. Cette contribution n'a pas pour objectif de comparer les différentes méthodes de traitement.

Le but de cette prise de position est de démontrer l'importance de la maladie variqueuse et des options thérapeutiques remboursables qui en découlent.

La phlébologie moderne a perfectionné la crossectomie classique associé au stripping et a également développé l'ablation thermique endoveineuse. Ces procédures endoluminales peu invasives par laser ou par radiofréquence sont effectuées dans la majorité des cas en ambulatoire ou en chirurgie de jour, et se caractérisent par une faible morbidité périopératoire et une grande sécurité. Les résultats à court et à long terme des méthodes opératoires et interventionnelles mentionnées ci-dessus montrent une efficacité comparable [4, 5]. Leur indication est basée sur les bilans anatomiques et physiopathologiques individuels [6-9]. Une évaluation individualisée du risque périopératoire et de l'indication d'un traitement en milieu hospitalier s'impose en cas de comorbidités importantes et de stades avancés d'insuffisance veineuse chronique (IVC) [10-12].

\section{Stades cliniques de l'insuffisance veineuse chronique}

L'évaluation clinique de l'insuffisance veineuse chronique s'appuie sur l'observation des lésions tissulaires provoquées par l'hypertension veineuse chronique au niveau des jambes. La classification CEAP (1995) [13] décrit l'étendue des lésions en six stades cliniques (C1-6):

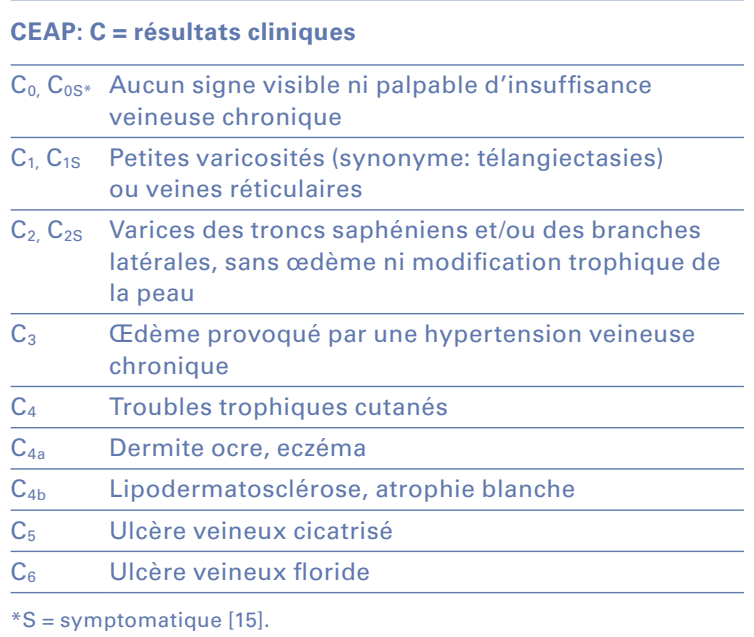

Dans environ la moitié des cas, la seule étiologie de l'insuffisance veineuse chronique est une insuffisance valvulaire du tronc saphénien et des branches latérales [14]

La gravité de l'insuffisance veineuse chronique n'est pas corrélée aux symptômes.

Le système veineux profond des jambes est continent et ne présente ni dommage ni reflux postthrombotiques. On peut traiter ces formes d'insuffisance veineuse chronique directement par l'ablation (stripping) ou destruction de ces veines (thermique ou chimique) [14]. 
Chez l'autre moitié des personnes présentant une insuffisance veineuse chronique, on trouve un reflux ou une occlusion dans le système veineux profond. Les occlusions correspondent à des séquelles de thrombose veineuse profonde non recanalisée. Une insuffisance veineuse superficielle secondaire se développe fréquemment par la suite $[6,7,13]$.

Les symptômes typiques ne sont toutefois pas spécifiques et peuvent se produire même en l'absence de varices.

La gravité de l'insuffisance veineuse chronique n'est pas corrélée aux symptômes. Malgré un stade avancé de la maladie, les symptômes peuvent rester modérés, voire totalement absents. Par conséquent, les varices peuvent également s'avérer être très prononcées et entraîner une insuffisance veineuse chronique, sans nécessairement provoquer de douleur. Les symptômes fréquents des varices comprennent: des douleurs s'étendant le long des trajets des veines atteintes, un prurit, la sensation de jambes lourdes, des fourmillements, des crampes ou des tensions. Ces symptômes typiques ne sont toutefois pas spécifiques et peuvent se produire même en l'absence de varices [15].

\section{Maladie variqueuse et thrombose veineuse des jambes}

Les varices augmentent le risque de thrombose veineuse superficielle des jambes (anciennement: thrombophlébite) [16]. Une thromboembolie veineuse symptomatique survient chez environ 6 à $24 \%$ des personnes atteintes de thrombose veineuse superficielle associée à une mortalité de $0,1 \%$. Des essais contrôlés randomisés ont montré une réduction significative de ce risque sous traitement par fondaparinux ou par rivaroxaban [17-19]. Le risque de thrombose veineuse superficielle est pratiquement inexistant après un traitement chirurgical ou interventionnel des varices.

\section{Bilan de l'IVC et des varices}

Le bilan de l'insuffisance veineuse chronique et de la varice s est basé sur l'examen clinique et l'examen anatomo-pathophysiologique au moyen de l'échographie Doppler [6-9]. L'examen ultrasonographique du système veineux (superficiel et profond) associé au Doppler est indispensable pour une bonne compréhension et planification du traitement [6-9].

Pour des questions spécifiques, on dispose également de techniques d'imagerie plus complexes, telles que la phlébographie classique, la phlébo-IRM ou le phlébo- scanner avec reconstruction 3D et les tests fonctionnels par pléthysmographie.

Le diamètre et le temps de reflux s'avèrent décisifs dans l'évaluation de la pertinence hémodynamique d'une varice. Un temps de reflux inférieur à 0,5 seconde pour un diamètre allant jusqu'à $3 \mathrm{~mm}$ a été défini comme normal par un consensus international [13].

\section{Indications, efficacité et limites du traitement par compression}

Le traitement par compression atténue temporairement les symptômes [20] sans action sur la cause, que ce soit sur les varices ou de l'insuffisance veineuse profonde $[6,7]$. La combinaison des traitements compressifs et invasifs des varices s'avère particulièrement efficace. En cas d'ulcère variqueux, parfait exemple d'insuffisance veineuse chronique avancée, les preuves en faveur de la combinaison d'un traitement par compression et d'un traitement chirurgical des varices sont particulièrement bien documentées, tant dans la prévention [21] que dans le traitement des ulcères veineux [22]. Même le port systématique de bas de contention à long terme ne peut remplacer un traitement opératoire ou interventionnel médicalement indiqué. En outre, il faut tenir compte du fait que le matériel médicalement prescrit pour la contention et les soins infirmiers fournis en cas de besoin engendrent des coûts sur de longue durée et bien supérieurs au traitement interventionel.

\section{Indications d'un traitement opératoire ou interventionnel des varices}

Les varices des troncs et des branches latérales de la grande et/ou de la petite veine saphène, ainsi que les varices non systématisées (non reliés clairement à un tronc ou une perforante), constituent une indication thérapeutique d'intervention chirurgicale [6-9]. Le choix entre la crossectomie avec stripping et une technique endoluminale (occlusion thermique par laser ou par radiofréquence) est guidé par l'anatomie et la physiopathologie du reflux veineux et des comorbidités [4-7].

\section{Indications de la sclérothérapie de la varicose (sclérothérapie écho- guidée à la mousse)}

L'ablation chimique des varices par injection de mousse sclérosante sous guidage échographique peut être pratiquée en ambulatoire et se révèle très efficace en cas d'indications appropriées, notamment: les récidives variqueuses diffuses et tortueuse (sauf sur des troncs saphéniens de gros calibre) [23], la varice isolée des 
branches latérales, ainsi que la présence de veines nourricières au voisinage d'ulcères veineux (feeder veins) [24].

\section{Traitements combinés}

Les acquis thérapeutiques des dernières décennies dans la prise en charge des varices permettent un traitement optimal par une combinaison des différentes techniques, soigneusement adaptée à chaque situation.

\section{Comorbidités pertinentes et risques périopératoires}

Les principales complications des traitements invasifs des varices sont spécifiques à chacune des procédures. Elles comprennent: les lymphœdèmes et lymphocèles, les lésions de nerfs sensibles, les hémorragies, les infections, les nécroses cutanées, les thromboses et les troubles de la cicatrisation des plaies [11, 12]. L'obésité représente l'un des facteurs de risque les plus fréquents

\section{Les principales complications des traitements invasifs des varices sont spécifiques à chacune des procédures.}

pour les troubles de la cicatrisation des plaies postopératoires, les nécroses cutanées et les infections [10]. Les récidives de varices inguinales de gros calibre, que l'on ne peut pas traiter par sclérothérapie écho-guidée à la mousse, comportent un risque accru de fistules lymphatiques et/ou de lymphœdème postopératoires [11]. Chez les personnes présentant en outre un diagnostic cardiovasculaire, il convient de peser soigneusement le pour et le contre de la cure chirurgicale des varices (réserve pour pontage veineux). En présence de facteurs de risque, la ou le spécialiste responsable du traitement doit décider, en fonction de leur gravité et de leur éventuelle combinaison, du choix de la procédure, voire de l'indication d'un traitement en milieu hospitalier.

\section{Impact médical et socio-économique de la maladie variqueuse}

La présence de varices étant socialement considérée comme «une vilaine tare», son traitement paraît d'emblée justifié d'un point de vue cosmétique. Cependant, les varices des jambes avec effet hémodynamique pathologique (stades cliniques C2-6) représente clairement une maladie à part entière: sans traitement, sa progression se poursuit inévitablement, entraînant des maladies secondaires et des complications qui surviennent avec une fréquence croissante. Environ 1\% des coûts de santé des systèmes sanitaires occidentaux résultent du traitement des plaies chroniques [25, 26]. Une prise en charge conséquente de la maladie variqueuse permettrait de réduire ce montant.

Le choix de la méthode de traitement doit être motivé individuellement, en fonction de l'anatomie, de la phy-

\section{Environ 1\% des coûts de santé des systèmes} sanitaires occidentaux résultent du traitement des plaies chroniques.

siopathologie et des comorbidités [6-9]. On dispose de deux procédures chirurgicales ou interventionnelles: la crossectomie avec stripping et l'ablation thermique endoveineuse [4-7]. La sclérothérapie à la mousse sous échographie duplex représente une bonne alternative thérapeutique, réalisable de manière simple et sûre dans des situations spécifiques, telles que dans le traitement de veines nourricières périulcéreuses (feeder veins), de varices atypiques (v. périnéales p. ex.) ou de la récidive de varices diffuses à la suite d'un traitement chirurgical [23, 24].

Il convient de distinguer ces traitements de celui des télangiectasies et veines réticulaires, à visée avant tout esthétique, à de rares exceptions près [3].

\section{Formation post-graduée}

Les phlébologues disposent (1) d'une attestation de formation complémentaire en phlébologie, (2) dans la plupart des cas, d'une attestation de formation complémentaire en ultrasonographie duplex du système veineux périphérique, et, (3) éventuellement, d'une attestation de formation complémentaire en ablation thermique endoveineuse (ATE). Les spécialistes en angiologie possèdent intrinsèquement ces deux premières qualifications et peuvent, s'il y a lieu, obtenir la troisième attestation de formation complémentaire. Historiquement, ce sont les les spécialistes en chirurgie vasculaire et spécialistes en chirurgie générale qui interviennent pour l'ablation des varices. Les spécialistes en chirurgie vasculaire peuvent obtenir l'attestation de formation complémentaire en ablation thermique endoveineuse de l'USSMV sans aucune condition, afin de compléter leur formation postgraduée, car leur programme de formation inclut une expertise en phlébologie et en ultrasonographie. Les spécialistes en chirurgie générale doivent en revanche satisfaire à certaines conditions.

L'Union des sociétés suisses des maladies vasculaires (composée de la Société suisse d'angiologie, de la Société suisse de phlébologie, de la Société suisse de chirurgie vasculaire et d'autres sociétés spécialisées) 
soutient sans réserve les efforts de politique sanitaire qui promeuvent l'utilisation efficace, appropriée et économique de la médecine moderne (critères EAE). Par conséquent, il va sans dire que les organismes de financement ne peuvent pas se permettre d'ignorer fondamentalement les indications médicales de la prise en charge des varices. Les assurées ont le droit d'accéder à une prise en charge optimale de la maladie variqueuse, autant au niveau du diagnostic précis qu'aux différentes techniques de son traitement. Cette position tient lieu de complément et de développement actuel à celle de la SSP, publiée par Kern et al. en 2004 [3].

\section{Références}

1 Rabe E, Pannier F, Bromen K, Schuldt K, Stang A, Poncar C, et al. Bonn Vein Study by German Society of Phlebology: Epidemiological study to investigate the prevalence and severity of chronic venous disorders in the urban and rural residential populations. Phlebologie. 2002;32:1-14.

2 Evans CJ, Fowkes FG, Ruckley CV, Lee AJ. Prevalence of varicose veins and chronic venous insufficiency in men and women in the general population: Edinburgh vein study. J Epidemiol Health. 1999;53:149-53.

3 Kern P, Ramelet AA, Küpfer S, Cassina P, Enzler M, Mahler F. Therapie der Varizen und Besenreiser der unteren Extremität: Medizinische und ästhetische Indikation. Schweiz Ärzteztg. 2004;85:2070-1

4 Rasmussen LH, Lawaetz M, Bjoern L, Vennits B, Blemings A, Eklof B. Randomized clinical trial comparing endovenous laser ablation, radiofrequency ablation, foam sclerotherapy and surgical stripping for greater saphenous veins. Br J Surg. 2011;98:1079-87.

5 Nesbitt C, Bedenis R, Bhattacharya V. Endovenous ablation (radiofrequency and laser) and foam sclerotherapy versus open surgery for great saphenous vein varices. Cochrane Database Syst Rev. 2014;cd005624

6 Gloviczki P, Comerota AJ, Dalsinf MC, Eklof BG, Gillespie DL, Gloviczki ML, et al.; Society of Vascular Surgery; American Venous Forum. The care of patients with varicose veins and associated chronic venous diseases: clinical practice guidelines of the Society for Vascular Surgery and the American Venous Forum. J Vasc Surg. 2011;53(Suppl):2S-48S.

7 Wittens C, Davies AH, Bækgaard N, Broholm R, Cavezzi A, Chastanet S, et al.: Management of Chronic Venous Disease. Clinical Practice Guidelines of the European Society for Vascular Surgery (ESVS). Eur J Vasc Endovasc Surg. 2015;49:678-737.

8 Spinedi L, Broz P, Engelberger RP, Staub D, Uthoff H. Clinical and duplex ultrasound evaluation of lower extremities varicose veins - a practical guideline. Vasa. 2017;46:325-36.

Dr Jürg Traber, médecin-chef Venenklinik Bellevue Kreuzlingen

Brückenstrasse 9

CH-8280 Kreuzlingen

j.traber[at]venenklinik.ch
9 García-Gimeno M, Rodríguez-Camarero S, Tagarro-Villalba S, Ramalle-Gomara, González-González E, González Arranz MA, et al. Duplex mapping of 2036 primary varicose veins. J Vasc Surg. 2009;49:681-9.

10 Tjeertes EK, Hoeks SE, Beks SB, Valentin TM, Hoofwijk AG, Stolker RJ. Obesity - a risk factor for postoperative complications in general surgery? BMC Anesthesiol. 2015;15:112.
11 Hayden A, Holdsworth J. Complications following re-exploration of the groin for recurrent varicose veins. Ann R Coll Surg Engl. 2001;83:272-3.

12 de Mik SML, Stubenrouch FE, Legemate, DA, Balm R, Ubbink DT, on behalf of the DISCOVAR study group. Treatment of varicose veins, international consensus on which major complications to discuss with the patient: A Delphi study. Phlebology. 2019;34:201-7.

13 Eklof B, Rutherford RB, Bergan JJ, Carpentier OH, Gloviczki P, Kistner RL, et al. Revision of the CEAP classification for chronic venous disorders: consensus statement. J Vasc Surg. 2004:40:1248-52

14 Shami SK, Sarin S, Cheatle TR, Scurr JH, Coleridge Smith PD. Venous ulcers and the superficial venous system. J Vasc Surg. 1993;17:487-90.

15 Blättler W, Mendoza E, Zollmann C, Bendix J, Amsler F. Homeostatic feelings - a novel explanation of vein symptoms derived from an experimental patient study. Vasa. 2019;48:492-501.

16 Zöller B, Ji J, Sundquist J, Sundquist K. Venous thromboembolism and varicose veins share familial susceptibility: a nationwide family study in Sweden. J Am Heart Assoc. 2014;26:3.

17 Decousus H, Prandoni P, Mismetti P, Bauersachs RM, Boda Z, Brenner B, et al. (CALISTO Study Group). Fondaparinux for the treatment of superficial-vein thrombosis in the legs. $\mathrm{N}$ Engl J Med. 2010;363:1222-32.

18 Beyer-Westendorf J, Schellong SM, Gerlach H, Rabe E, Weitz JI, Jersemann K, et al. Prevention of thromboembolic complications in patients with superficial-vein thrombosis given rivaroxaban or fondaparinux: the open-label, randomised, noninferiority SURPRISE phase $3 \mathrm{~b}$ trial. Lancet Haematol. 2017;4:e105-e113.

19 Decousus H, Quere I, Presles E, et al. Superficial venous thrombosis and venous thromboembolism: a large, prospective epidemiologic study. Ann Intern Med. 2010;152:218-24.

20 Rabe E, Partsch H, Hafner J, Lattimer C, Mosti G, Neumann M, et al. Indications for medical compression stockings in venous and lymphatic disorders: an evidence-based consensus statement. Phlebology. 2018;33(3):163-84.

21 Barwell JR, Davies CE, Deacon J, Harvey K, Minor J, Sassano A, et al. Comparison of surgery and compression with compression alone in chronic venous ulceration (ESCHAR study): randomized controlled trial. Lancet. 2004;363(9424):1854-9.

22 Gohel MS, Heatley F, Liu X, Bradbury A, Bulbulia R, Cullum N, et al. (EVRA Trial Investigators). A randomized controlled trial of early endovenous ablation in venous ulceration. $\mathrm{N}$ Engl J Med. 2018;378:2105-14.

23 Darvall KAL, Bate GR, Adam DJ, Silverman SH, Bradbury AW. Duplex ultrasound outcomes following ultrasound-guided foam sclerotherapy of symptomatic recurrent great saphenous varicose veins. Eur J Vasc Endovasc Surg. 2011;42:107-14.

24 Kulkarni SR, Slim FJA, Emerson LG, Davies C, Bulbulia RA, Whyman MR, et al. Effect of foam slcerotherapy on healing and long-term recurrence in chronic venous leg ulcers. Phlebology. 2013;28:140-6.

25 Müller-Bühl U, Leutgeb R, Bungartz J, Szecsenyi J, Laux G. Expenditure of chronic venous leg ulcer management in German primary care: results from a population-based study. Int Wound J. 2013;10:52-6.

26 Rice JB, Desai U, Cummings AK, Birnbaum HG, Skornicki M, Parsons N. Burden of venous leg ulcers in the United States. J Med Econ. 2014;17:347-56 